\title{
miR-137 Controls Proliferation and Differentiation of Human Adipose Tissue Stromal Cells
}

\author{
Keun Koo Shin ${ }^{a, b, c}$ Young Suk Kimª, Jee Young Kimª,b Yong Chan Bae ${ }^{\mathrm{d}}$ \\ Jin Sup Jung ${ }^{a, b, c, e}$ \\ ${ }^{a}$ Department of Physiology, School of Medicine, Pusan National University, ${ }^{b}$ Medical Research Center \\ for Ischemic Tissue Engineering, Pusan National University, 'BK21 Medical Science Education Center, \\ School of Medicine, Pusan National University, Yangsan, dDepartment of Plastic Surgery, School of \\ Medicine, Pusan National University, eMedical Research Institute, Pusan National University, Pusan, \\ Korea
}

\section{Key Words}

MicroRNA • hADSC • Adipogenic differentiation • Proliferation • miR-137

\begin{abstract}
Background/Aims: Demonstrating the molecular mechanisms of human adipose tissuederived mesenchymal stem cells (hADSCs) differentiation and proliferation could develop hADSCs-based cell therapy. Methods: The microRNA-137 (miR-137) and cell division control protein 42 homolog (CDC42) levels were regulated by oligonucleotides transfection. The adipogenic differentiation was induced for 10 days in an adipogenic medium and assessed by using an Oil Red O stain. The regulation of miR-137 on CDC42 expression was determined by western blot, real-time PCR and luciferase reporter assay. Results: We confirmed the roles of miR-137 on hADSCs proliferation and adipogenic differentiation. We showed that overexpression of miR-137 inhibited both hADSCs proliferation and adipogenic differentiation. Overexpression of miR-137 also downregulated protein and mRNA levels of CDC42, a predicted target of miR-137. In contrast, inhibition of miR-137 with 2'-O-methyl antisense RNA increased proliferation and adipogenic differentiation in hADSCs. Luciferase reporter activity in the miR-137 target site within the CDC42 3'UTR was lower in miR-137-transfected hADSCs than in control miRNA-transfected hADSCs. RNA interference-mediated downregulation of CDC42 in hADSCs inhibited their proliferation and adipogenic differentiation. Conclusion: Our results indicate that miR-137 regulates hADSCs adipogenic differentiation and proliferation by directly targeting $C D C 42$. These findings improve our knowledge of the molecular mechanisms governing hADSCs differentiation and proliferation.
\end{abstract}




\section{Introduction}

MicroRNAs (miRNAs) are endogenous 19- to 25-nucleotide non-coding RNAs that bind to partially complementary recognition sequences of mRNA, resulting in translational repression or cleavage and degradation of mRNA, thus regulating the expression of miRNA targets [1]. Furthermore, each miRNA can regulate multiple mRNAs and each mRNA can be targeted by a number of miRNAs. Thus, it is evaluated that as high as $30 \%$ of protein-coding genes could be targets of miRNAs. miRNAs have been involved in diverse biological processes including differentiation [2], proliferation, apoptosis [3, 4], metabolism [3] and neuronal patterning [5]. miRNAs play important roles in the self-renewal and pluripotency of stem cells $[6,7]$. The functions of miR-137 have been mostly investigated in several cancer cells. In glioblastoma [8-11], oral squamous cell carcinoma [12], lung cancer [13], colorectal cancer $[14,15]$ and gastric cancer [16], miR-137 either induced cell cycle arrest in G1 or inhibited invasion through interaction with various target molecules. MiR-137 plays a significant role in the neuronal maturation and dendritic morphogenesis of young neurons [8, 17]. miR-137 is one of the two miRNAs in embryonic stem cells (ESCs) that are co-occupied by the key transcription factors: Oct4, Sox2, and Nanog [18], and is mainly expressed in embryonic stem cells at mitotic stages of the cell cycle. The expression of miR-137 is significantly upregulated when ESCs [19] and neural stem cells [20,21] are differentiated into neuronal lineage.

Adipose tissue, like bone marrow, is a mesodermally-derived organ with a stromal cell population [22]. This population, termed adipose tissue-derived mesenchymal stem cells or adipose tissue stromal cells (ADSCs), shares many of its characteristics with bone marrow. These characteristics include extensive proliferative potential and the ability to differentiate toward adipogenic, osteogenic, chondrogenic and myogenic lineages [23-25]. Understanding the molecular events involved in adipocyte differentiation is interesting for the development of therapeutics for metabolic diseases such as obesity and diabetes. Our laboratory reported that several microRNAs control proliferation and differentiation of human adipose tissuederived mesenchymal stem cells (hADSCs) $[26,27]$.

In this study, we examined the role of miR-137 on proliferation and adipogenic differentiation of hADSCs. Our data showed that miR-137 inhibits hADSCs proliferation and adipogenic differentiation by binding to specific target sequences harbored in the 3' untranslated region (UTR) of cell division control protein 42 homolog (CDC42) mRNA.

\section{Materials and Methods}

\section{Cell culture}

All protocols involving human subjects were approved by the Institutional Review Board of Pusan National University. Superfluous materials were collected from four individuals undergoing elective abdominoplasty after informed consent was given by each individual. The hADSCs were isolated according to the methods described in previous studies [28]. hADSCs were maintained in Low-glucose DMEM with $10 \%$ fetal bovine serum, $100 \mu \mathrm{g} / \mathrm{ml}$ streptomycin and $100 \mathrm{mg} / \mathrm{ml}$ penicillin in $5 \% \mathrm{CO}_{2}$ environment at $37^{\circ} \mathrm{C}$.

\section{Induction of differentiation}

Adipogenic differentiation was induced by culturing ADSCs for 10 days in an adipogenic medium $(10 \%$ FBS, $1 \mu \mathrm{M}$ dexamethasone, $0.5 \mathrm{mM} / \mathrm{mL}$ 3-isobutyl-1-methylxanthine, and $200 \mu \mathrm{M}$ indomethacin in $\alpha$-MEM) and assessed by the use of an Oil Red $O$ stain as an indicator of intracellular lipid accumulation. In order to obtain quantitative data, $1 \mathrm{~mL}$ of isopropyl alcohol was added to the stained culture dish. The dye was extracted into a 96-well plate and the absorbance read in a plate reader at $562 \mathrm{~nm}$.

\section{Evaluation of cell proliferation}

hADSCs were transfected with miRNA or siRNA. After a $48 \mathrm{hr}$ incubation, the cells were detached using Hank's balanced salt solution containing $0.05 \%$ trypsin and $0.02 \%$ EDTA, then plated in 6 well plates at a density of $1 \times 10^{4}$ cells/well. The number of cells was counted using a Countess ${ }^{\circledR}$ Automated Cell Counter (Invitrogen, Carlsbad, CA, USA). 
Real-time polymerase chain reaction (PCR)

Total RNA was extracted using Trizol (Invitrogen), according to the manufacturer's instructions and reverse-transcribed into cDNA with the Reverse Transcriptase M-MLV (Promega, Madison, WI, USA). Internal control for expression analysis was GUSB (Beta-D-glucuronidase). Primer sequences to be used in the experiment were as follows: CDC42 FW, 5'-ATC GGA ATA TGT ACC GAC TG-3' and RV, 5'-GTC TTT GGA CAG TGG TGA GT-3'; GUSB FW, 5'-GCG TCC CAC CTA GAA TCT GC-3' and RV, 5'-CAT ACG GAG CCC CCT TGT C-3'; aP2 (fatty acid binding protein) FW, 5'-GGT GGT GGA ATG CGT CAT G-3' and RV, 5'-CAA CGT CCC TTG GCT TAT GC-3'; PPAR $\gamma$ (peroxisome proliferator-activated receptor gamma) FW, 5'-GAG CCC AAG TTT GAG TTT GC-3' and RV, 5'-CTG TGA GGA CTC AGG GTG GT-3'; PPAR 2 2 FW, 5'-CCA TGC TGT TAT GGG TGA A-3' and RV, 5'-TGG GAG TGG TCT TCC ATT A-3'; C/EBP- $\alpha$ (CCAAT/enhancer binding protein alpha) FW, 5'-GCA AGG CCA AGA AGT CGG TGG AC-3' and RV, 5'-TGC CCA TGG CCT TGA CCA AGG AG-3'. For each primer pair, the linearity of detection was confirmed by measuring a dilution curve with cDNA isolated from hADSCs. GUSB mRNA, of which expression was maintained at a constant levels during differentiation, was amplified as an internal control. The data were presented as the ratio of a target gene level to GUSB level of each sample.

For miRNA quantitative RT-PCR (qPCR), small RNA species enriched RNA isolation was performed as per the manufacturer's instructions (mirVana miRNA isolation kit; Ambion, Austin, TX, USA). miRNA was reverse-transcribed according to the manufacturer's specified guidelines, using Ncode miRNA firststrand cDNA synthesis kits (Invitrogen). Forward primer sequence was designed as the corresponding mature miRNA sequences and 5S rRNA was used as normalizing control. The miR-137-specific forward primer sequence was designed on the basis of miRNA sequences obtained from the miRBase database. Real time PCR was performed using a Power SYBR Green PCR Master Mix on the ABI 7500 Instrument (Applied Biosystems, Warrington, UK).

\section{Western blot analysis}

Samples were homogenized in RIPA buffer (Sigma, St. Louis, MO, USA). The isolated proteins were separated by SDS-PAGE and electro-transferred to PVDF membranes (Millipore, Bedford, MA, USA). Blots were probed with primary antibodies, followed by HRP-conjugated secondary antibodies. Antibodies used in this study were purchased as follows: CDC42 from Abcam (Cambridge, MA); GAPDH from Cell Signaling Technologies (Boston, MA). Bound antibodies were detected using an ECL detection kit (Pierce Biotechnology, Rockford, IL, USA) and visualized using LAS 3000 Luminoimage Analyzer (Fujifilm, Tokyo, Japan). Protein level was quantified using the National Institutes of Health ImageJ software.

\section{miRNA / siRNA transfection}

hADSCs were seeded with complete medium without antibiotics. On the following day, 20nM of miRNAs (miR-137 mimics or inhibitors, Dharmacon, Thermo Scientific, Epsom, UK) and 100nM of siRNA (on-TARGET plus SMART pool, Dharmacon) for CDC42 were transfected using DharmaFECT Transfection Reagent I (Dharmacon) according to manufacturer's protocol with miRNA negative control or non-targeting siRNA as a negative control.

\section{Reporter Vectors and DNA Constructs}

A putative miR-137-recognition element (as single copy) from the CDC42 gene was cloned in the 3 '-untranslated region (UTR) of the firefly luciferase reporter vector according to the manufacturer's specified guidelines. The oligonucleotide sequences were designed to carry the HindIII and SpeI sites at their extremities facilitating ligation into the HindIII and SpeI sites of pMIR-Report (Ambion). The oligonucleotides used in these studies were: pMIR-CDC42 FW, 5'-CTAGT TTAAAATTCGTTTTTGCAATAAT A-3' and RV, 5 '-AGCTT ATTATTGCAAAAACGAATTTTAA A-3'; pMIR-CDC42-mut FW, 5'-CTAGT TTAAAATTCGTTTTTCGTTATTT A-3' and RV, 5'-AGCTT AAATAACGAAAAACGAATTTTAA A -3'.

\section{Reporter gene assay}

All transient transfections were conducted using Lipofectamine Plus Reagent (Invitrogen). The pMIRCDC42 and pMIR- $\beta$-gal plasmids were used as reporter constructs. The cells were harvested $48 \mathrm{hr}$ after transfection, lysed in RLB buffer, and subsequently assayed for their luciferase activity (Luciferase Assay System, Promega). The transfections were performed in duplicate, and all experiments were repeated several times. The luciferase assays were normalized according to their $\beta$-galactosidase activity. 


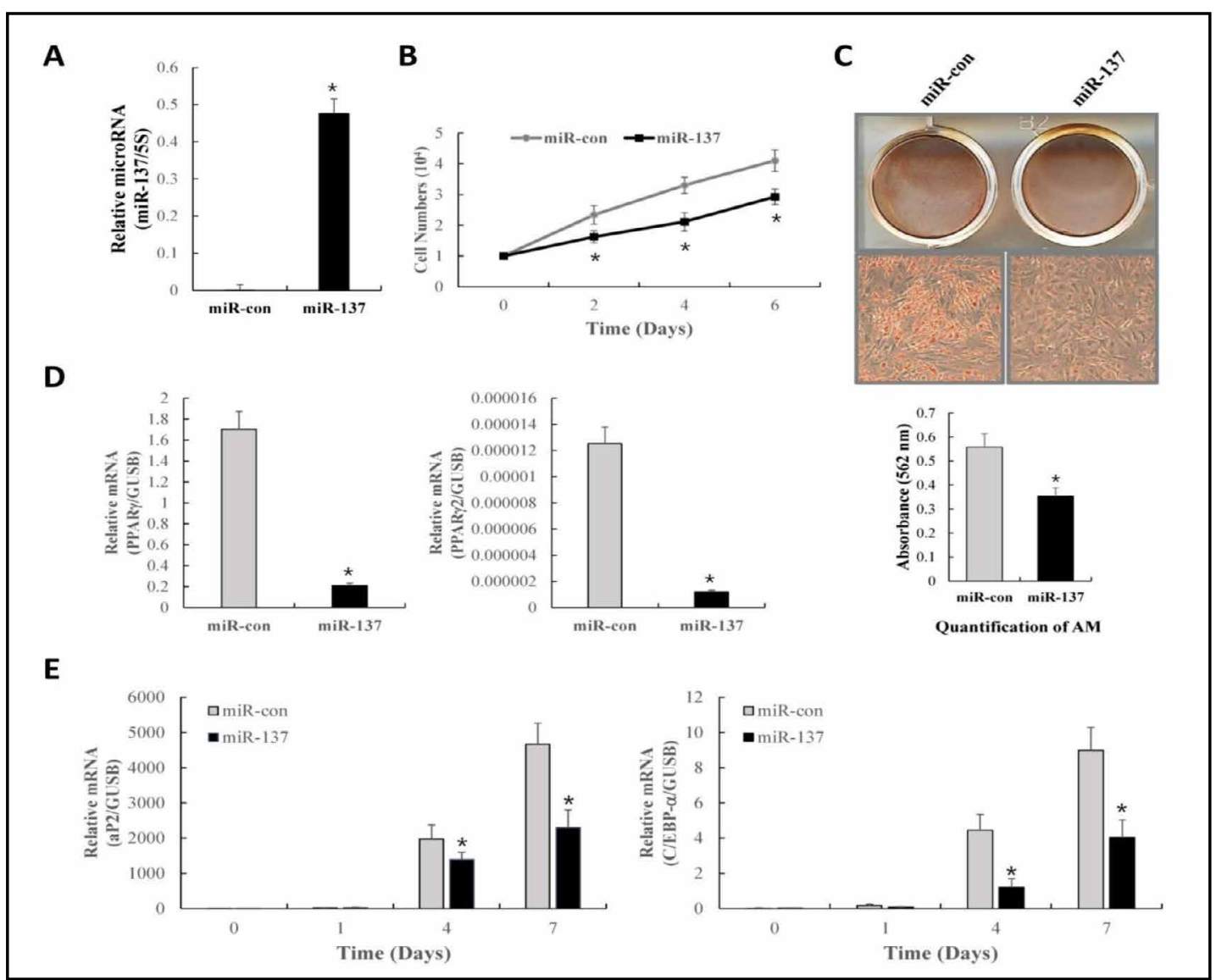

Fig. 1. Overexpression of miR-137 inhibits adipogenic differentiation and proliferation of hADSCs. (A) miR137 levels were determined in control- (miR-con) or miR-137-transfected hADSC using real-time PCR. The data were presented as the relative ratio of miR-137 to 5S RNA level of each sample. (B) hADSC proliferation was determined by direct cell counting after oligonucleotide transfection. (C) Oligonucleotide-transfected hADSC were grown to 80-90\% confluence. Adipogenic differentiation was induced for 10 days and determined by Oil Red 0 staining to visualize intracellular lipid accumulation, which was quantified by absorbance at 562nm. (D and E) Real-time PCR analysis of PPAR $\gamma$ and PPAR 2 in miR-137 mimic-transfected undifferentiated cells (D). Changes in expression of $a P 2$ and $C / E B P-\alpha$ in differentiated cell to adipogenic lineage (E). Total RNA was isolated at days after induction of differentiation. Data represent mean \pm SEM of the ratio of a target gene level to GUSB level $(n=4)$. ${ }^{*} p<0.05$ compared with miR-con-transfected hADSCs.

\section{Statistical analysis}

All of the results are presented as the means \pm SEM. Comparisons between groups were analyzed via t-tests (2-sided) or ANOVA for experiments with more than 2 subgroups. Post hoc range tests and pairwise multiple comparisons were conducted using the t-test (2-sided) with Bonferroni adjustments. Probability values of $\mathrm{P}<0.05$ were considered to be statistically significant.

\section{Result}

Effects of miR-137 on the adipogenic differentiation and proliferation of hADSC

We chose the microRNAs that showed high levels of expression in preliminary microRNA microarray study and then targeted differentiation-related molecules using miRWalk database [29]. To examine the role of miR-137 in hADSCs functions, we transfected $20 \mathrm{nM}$ of miR-137 mimics into hADSCs for the overexpression of miR-137. miRNA mimics augments 
A

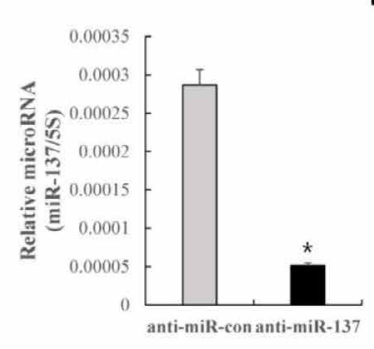

D

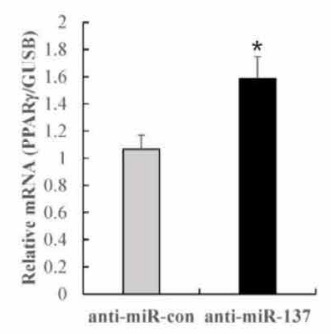

$\mathbf{E}$

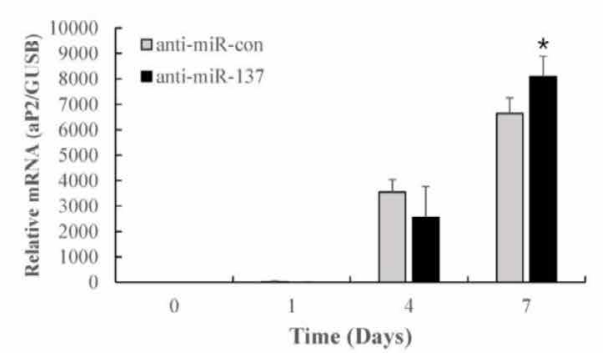

B
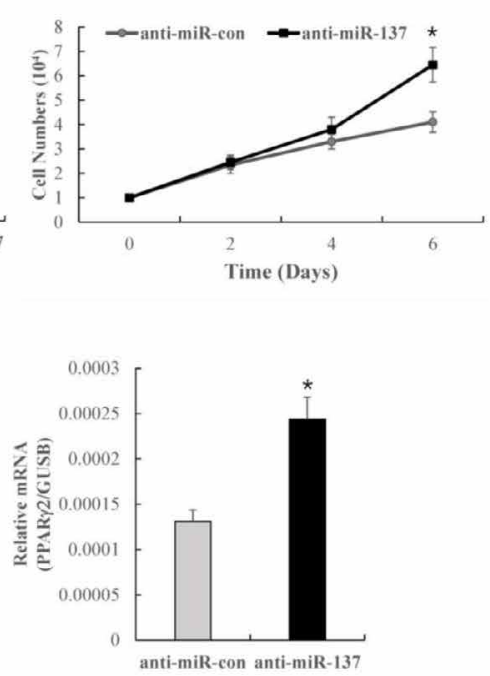

C
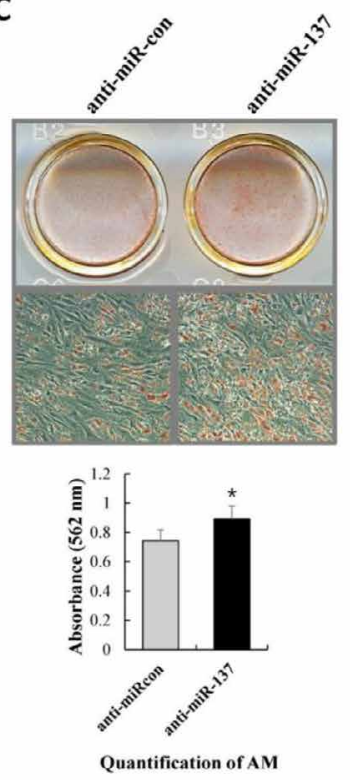

Fig. 2. Inhibition of miR-137 increases adipogenic differentiation and proliferation of hADSCs. (A) miR137 levels were determined in control- (anti-miR-con) or anti-miR-137-transfected hADSC using real-time PCR. The data were presented as the relative ratio of miR-137 to 5S RNA level of each sample. (B) hADSC proliferation was determined by direct cell counting after oligonucleotide transfection. (C) Oligonucleotidetransfected hADSC were grown to $80-90 \%$ confluence. Adipogenic differentiation was induced for 10 days and determined by Oil Red 0 staining to visualize intracellular lipid accumulation, which was quantified by absorbance at $562 \mathrm{~nm}$. (D and E) Real-time PCR analysis of PPAR $\gamma$ and PPAR 2 in anti-miR-137-transfeted undifferentiated cells (D). Changes in expression of $a P 2$ and $C / E B P-\alpha$ in differentiated cell to adipogenic lineage (E). Total RNA was isolated at days after induction of differentiation. Data represent mean \pm SEM of the ratio of a target gene level to GUSB level $(n=4) .{ }^{*} p<0.05$ compared with anti-miR-con transfected hADSCs.

the function of endogenous miRNAs for easier detection of a phenotypic change. Real-time PCR analysis showed that miR-137-transfected hADSCs increased miR-137 expression (Fig. 1A).

To test the effect of miR-137 on hADSCs proliferation, we plated miR-137-transfected hADSCs $\left(1 \times 10^{4}\right.$ cells/well $)$ on culture plates and counted the number. Cell counting on the days indicated in Fig. 1B showed that miR137-transfected cells had slower growth than control cells. We induced adipogenic differentiation of miR-137-overexpressing hADSCs with the appropriate media. After incubating hADSCs with adipogenic media for 10 days, miR137-overexpressing hADSCs significantly inhibited adipogenic differentiation. The inhibition was quantified by absorbance of Oil Red $\mathrm{O}$ staining (Fig. 1C). We then used real-time PCR 


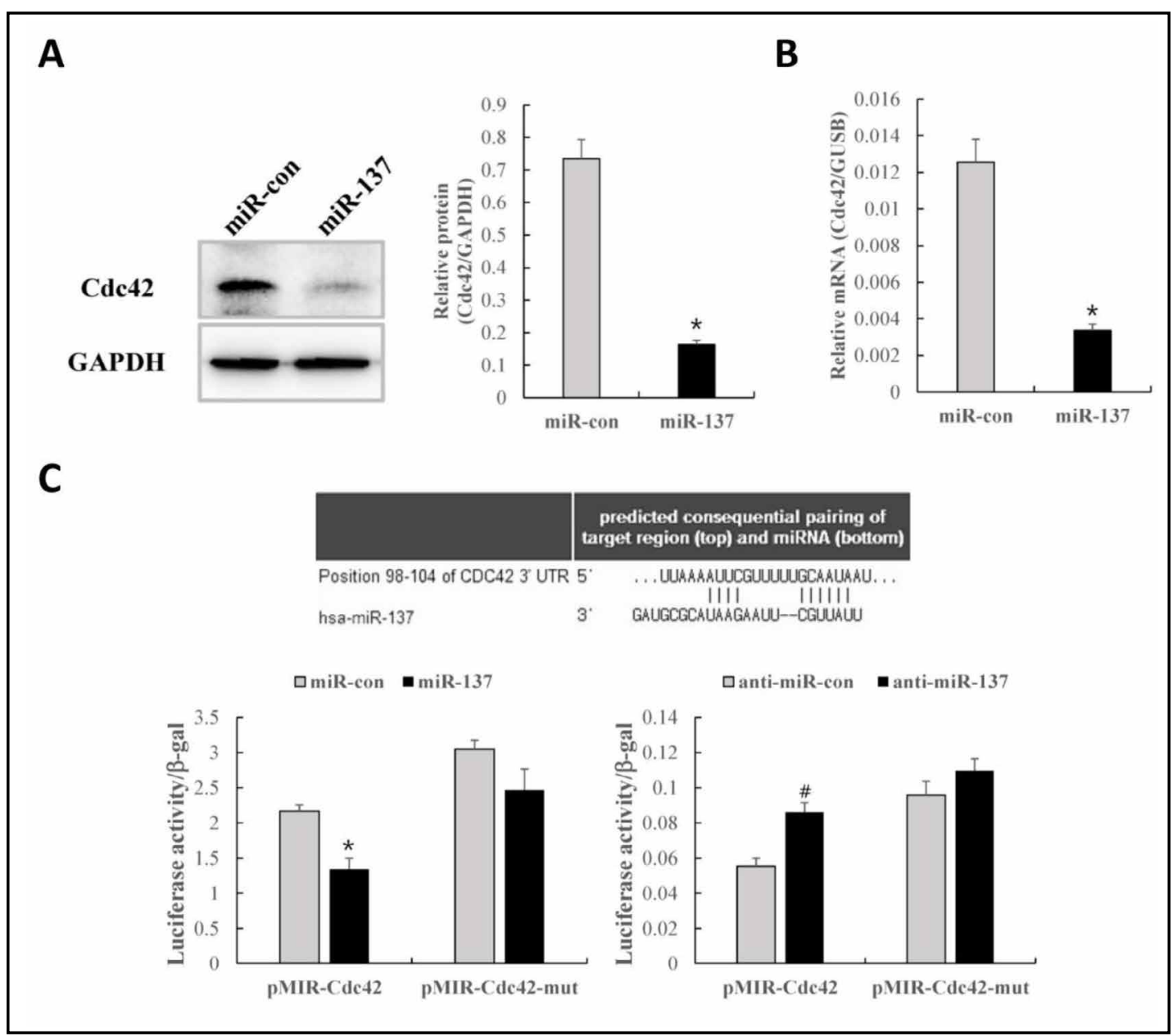

Fig. 3. miR-137 targets the 3'UTR of CDC42 mRNA. (A, B) CDC42 expression in hADSCs transfected with oligonucleotide was analyzed by western blot (A) and real-time PCR (B). (C) pMIR-CDC42 or pMIR-CDC42-mut luciferase constructs were transfected into miR-con or miR-137-mimic-transfected hADSCs and anti-miRcon or anti-miR-137-transfected hADSCs. Data represent mean \pm SEM of the ratio of a target gene level to GUSB level $(n=4),{ }^{*} p<0.05$, compared with miR-con-transfected hADSCs, ${ }^{*} p<0.05$ compared with antimiR-Con-transfected hADSCs.

to determine whether miR-137 overexpression decreased the expression of adipogenesisrelated genes. The results showed that miR-137-overexpressing hADSCs revealed lower levels of PPAR $\gamma$ and PPAR $\gamma$ expression than control microRNA-transfected cells (Fig. 1D). Real time PCR analysis of differentiated cells showed that the transfection of miR-137 mimic inhibited $a P 2$ and $C / E B P-\alpha$ expression at 4 days and 7 days after differentiation (Fig. 1E).

\section{Effect of miR-137 inhibition on the differentiation and proliferation of hADSCs}

To investigate the effect of miR-137 inhibition on hADSCs differentiation, we transfected hADSCs with a specific miRNA inhibitor (anti-miR-137). Real-time PCR analysis showed that transfection of anti-miR-137 effectively downregulated miR-137 expression in hADSCs (Fig. 2A). Cell number counts showed that anti-miR-137 enhanced hADSCs proliferation compared with control oligonucleotide-transfected cells (Fig. 2B).

To examine the effect of miR-137 inhibition on adipogenic differentiation, anti-miR-137transfected hADSCs were induced to differentiate into adipogenic lineages. Oil red 0 staining revealed that the inhibition of miR-137 enhanced adipogenic differentiation of hADSCs (Fig. 2C). To further confirm the effect, we determined adipogenic marker genes expression by 
Fig. 4. $C D C 42$ RNAi inhibits adipogenic differentiation and proliferation of hADSC. (A) CDC42 mRNA levels were determined in control- (sicon) or CDC42 oligonucleotide- (si-CDC42) transfected hADSC using real-time PCR. (B) hADSC proliferation was determined by direct cell counting after siRNA oligo transfection. (C) siRNA oligo-transfected hADSC were grown to $80-90 \%$ confluence. Adipogenic differentiation was induced for 10 days and determined by Oil Red 0 staining to visualize intracellular lipid accumulation, which was quantified by absorbance at $562 \mathrm{~nm}$. Silencing of the CDC42 gene inhibited the adipogenic differentiation of hADSCs. (D) Real-time PCR analysis of PPAR 2 and $C / E B P-\alpha$ in si-CDC42-transfeted undifferentiated cells. Data represent mean \pm SEM of the ratio of a target gene level to GUSB level ( $n=4)$. ${ }^{*} \mathrm{p}<0.05$ compared with sicon-transfected hADSCs.

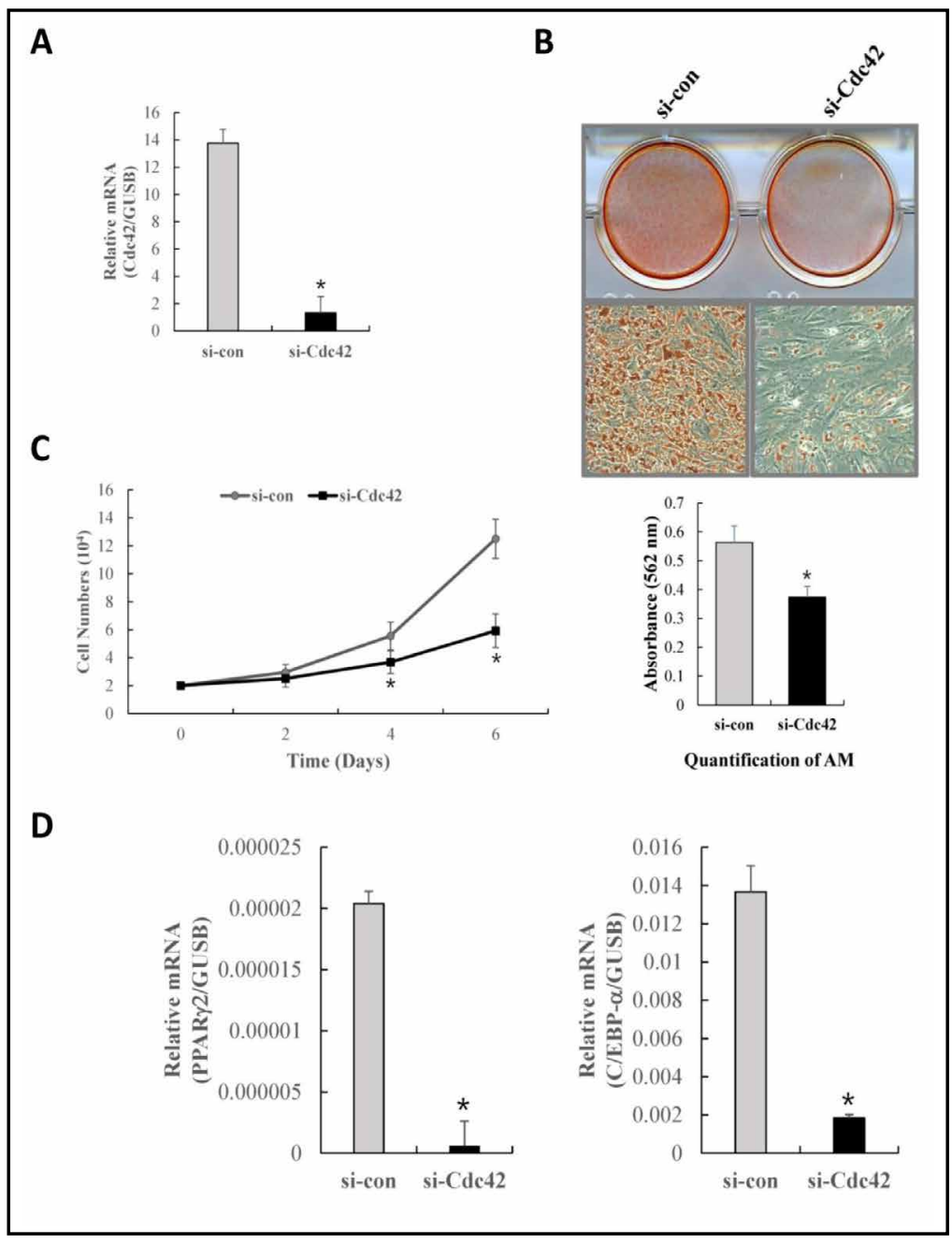

real-time PCR. Anti-miR-137 transfection increased the expression of marker genes (PPAR $\gamma$ $P P A R \gamma 2, a P 2$ and $C / E B P-\alpha$ ) during adipogenic differentiation of hADSCs (Figs. 2D and 2E).

miR-137 targets the 3'UTR of CDC42 mRNA

To determine the potential targets of miR-137 in hADSCs, we analyzed the miR-137induced changes in gene expression profile by microarray. Using miRWalk database for target gene prediction, $C D C 42$ in genes where expression was downregulated in microarray analysis was selected as the potential target of miR-137.

To determine the relationship between miR-137 and CDC42, we analyzed CDC42 expression with real time PCR and western blot analysis in miR-137 mimic transfected cells. The transfection of miR-137 mimic led to a significant decrease in CDC42 expression at both protein (Fig. 3A) and mRNA levels (Fig. 3B) in the hADSCs.

To test whether miR-137 directly targeted $C D C 42$ in hADSCs, we constructed luciferase reporter genes with $C D C 42$ 3'UTR with or without mutation at the miR-137 binding regions and confirmed their expression with miR-137 transfection. The results showed that a decrease in relative luciferase activity was demonstrated when the CDC42 3'UTR (pMIRCDC42) was transfected into miR-137-transfected hADSCs, while no decrease in relative luciferase activity was observed in hADSCs transfected with mutant CDC42 3'UTR (pMIRCDC42-mut). On the contrary, transfection of anti-miR-137 increased the luciferase activity of pMIR-CDC42 in hADSCs compared with transfection of the miRNA control (Fig. 3C). 
Effect of CDC42 on adipogenic differentiation and proliferation of hADSCS

To determine the role of $C D C 42$ in adipogenic differentiation and proliferation of hADSCs, we suppressed CDC42 expression in hADSCs with an RNA interference technique using $C D C 42$ siRNA (si-CDC42) transfection. Real-time PCR analysis confirmed that si-CDC42 effectively inhibited $C D C 42$ expression in hADSCs (Fig. 4A). We also determined the effect of CDC42 downregulation on hADSCs proliferation. Direct cell counting showed that si-CDC42transfected hADSCs proliferated less than the control cells (Fig. 4B).

To further study the effect of CDC42 downregulation on the efficiency of hADSCs differentiation, we induced adipogenic differentiation of si-CDC42-transfected hADSCs. Oil red 0 staining and real-time PCR analysis of adipogenic marker genes indicated that downregulation of CDC42 inhibited adipogenic differentiation of hADSCs (Figs. 4C and 4D).

\section{Discussion}

While several reports have demonstrated the function of miR-137 in neurogenesis within ESCs $[19,20]$, no studies to date have examined the effects on other lineage differentiation. In the present study, we investigated whether miR-137 was involved in proliferation and adipogenic differentiation of hADSCs. We demonstrated that miR-137 overexpression with miR-137 mimic in hADSCs inhibited proliferation and adipogenic differentiation. We also demonstrated and that miR-137 inhibition by transfection of inhibitor oligonucleotids increased adipogenic differentiation and proliferation. These results indicated that miR-137 negatively regulated adipogenic differentiation and proliferation in hADSCs.

Adipogenesis is a very complex process significantly involved in diseases including obesity, insulin resistance, and type 2 diabetes [30,31]. It is regulated by multiple transcription factors, especially the CCAAT/enhancer-binding protein $(C / E B P)$ family and peroxisome proliferator-activated receptor $\gamma(P P A R \gamma)[32,33]$. PPAR $\gamma$ is certainly necessary for the adipogenic differentiation in in vivo models [34]. In this study we showed that the modulation of miR-137 level altered the expression of PPAR gamma in undifferentiated hADSCs.

We identified from microarray experiment that one of the downregulated genes in miR-137 mimic-transfected cells is CDC42. The data in this experiment showed that overexpression of miR-137 downregulated the expression of CDC42 at the protein and mRNA levels. An assay with a firefly luciferase reporter plasmid containing the predicted CDC42 target gene sequences showed that overexpression of miR-137 decreased luciferase activity and inhibition of miR-137 increased luciferase activity, confirming the direct binding of miR-137 to 3'UTR of CDC42 in hADSCs. The role of miR-137 in the proliferation and adipogenic differentiation of hADSCs was further supported by determining the effect of CDC42 downregulation. In this study, the downregulation of CDC42 using RNA interference inhibited the adipogenic differentiation and proliferation of hADSCs (Fig. 4)

$C D C 42$, as a key member of the Rho GTPase family, is well known to be involved in proliferation, migration, invasion, apoptosis and angiogenesis, in several types of cancers. CDC42 contributes to the regulation of cell cycle progression in G1/S-phase transition by activation of downstream molecules such as ERK1/2 and cyclin D1 [35]. Consistent with our findings, miR-137 has been reported to regulate cell proliferation and invasion by targeting CDC42 gene in several cancers $[13,14,16]$.

Although the role of $C D C 42$ in cell fate determination has been reported in neural progenitor cells [36], epidermal stem cells [37] and hematopoietic stem cells [38], the relationship between $C D C 42$ and adipogenic differentiation has not been reported. In spite of high sequence analogies among Rho GTPase family members, many Rho GTPases play functionally unique and essential roles, and these roles are often cell type specific. In this study we showed that miR-137 inhibits adipogenic differentiation through inhibition of CDC42 function. The Rho GTPase family members involved in adipogenesis have shown various actions according to subtypes. Sordella et al [39] reported that activation of Rho inhibits adipogenesis in Mouse embryonic fibroblasts (MEFs) or 3T3-L1 cells, while Mcbeath 
et al [40] showed that cell shape changes induced by expression of dominant-negative RhoA committed human mesenchymal stem cells (hMSCs) to become adipocytes. Rho guanine nucleotide exchange factors (RhoGEFs), which stimulate the exchange of GDP for GTP in order to generate the activated form of GTPases and activate RhoA, CDC42 and Rac1, inhibits adipogenesis in 3T3-L1 cells [41]. In contrast, Nishizuka et al [42] reported that the overexpression of TC10-like/TC10_Long, a member of Rho GTPase, induced adipogenic differentiation in the presence of the ligand for PPAR gamma in NIH3T3 cells. Differential effects of Rho family members on cellular functions also have been reported in mouse fibroblast [43], intestinal epithelial cells [44] and hematopoietic cells [45].

miR-137 that regulates adipogenic differentiation and proliferation of hADSCs through the modulation of CDC42 levels can provide mechanistic insights into the molecular processes of stem cell differentiation and proliferation. These findings present miR-137 as a potential target for adipose tissue engineering through miRNAs and in the management of obesity and other metabolic diseases.

\section{Conflict of interests}

The authors declare no conflict of interest.

\section{Acknowledgements}

This work was supported by the National Research Foundation of Korea(NRF) grant funded by the Korea government(MSIP) (2012M3A9B4028558) and the MRC program of MEST/KOSEF (2007-0052078).

\section{References}

1 Bartel DP: MicroRNAs: genomics, biogenesis, mechanism, and function. Cell 2004;116:281-297.

2 Tarantino C, Paolella G, Cozzuto L, Minopoli G, Pastore L, Parisi S, Russo T: miRNA 34a, 100, and 137 modulate differentiation of mouse embryonic stem cells. FASEB J 2010;24:3255-3263.

3 Xu P, Vernooy SY, Guo M, Hay BA: The Drosophila microRNA Mir-14 suppresses cell death and is required for normal fat metabolism. Curr Biol 2003;13:790-795.

-4 Brennecke J, Hipfner DR, Stark A, Russell RB, Cohen SM: bantam encodes a developmentally regulated microRNA that controls cell proliferation and regulates the proapoptotic gene hid in Drosophila. Cell 2003;113:25-36.

5 Johnston RJ, Hobert O: A microRNA controlling left/right neuronal asymmetry in Caenorhabditis elegans. Nature 2003;426:845-849.

6 Liu C, Tang DG: MicroRNA regulation of cancer stem cells. Cancer Res 2011;71:5950-5954.

$\checkmark 7$ Pauli A, Rinn JL, Schier AF: Non-coding RNAs as regulators of embryogenesis. Nat Rev Genet 2011;12:136149.

$\checkmark 8$ Silber J, Lim DA, Petritsch C, Persson AI, Maunakea AK, Yu M, Vandenberg SR, Ginzinger DG, James CD, Costello JF, Bergers G, Weiss WA, Alvarez-Buylla A, Hodgson JG: miR-124 and miR-137 inhibit proliferation of glioblastoma multiforme cells and induce differentiation of brain tumor stem cells. BMC Med 2008;6:14.

-9 Althoff K, Beckers A, Odersky A, Mestdagh P, Köster J, Bray IM, Bryan K, Vandesompele J, Speleman F, Stallings RL, Schramm A, Eggert A, Sprüssel A, Schulte JH: MiR-137 functions as a tumor suppressor in neuroblastoma by downregulating KDM1A. Int J Cancer 2013;133:1064-1073.

-10 Li KK, Yang L, Pang JC, Chan AK, Zhou L, Mao Y, Wang Y, Lau KM, Poon WS, Shi Z, Ng HK: MIR-137 suppresses growth and invasion, is downregulated in oligodendroglial tumors and targets CSE1L. Brain Pathol 2013;23:426-439. 
Shin/Kim/Kim/Bae/Jung: Role of miR-137 in hADSC

-11 Bier A, Giladi N, Kronfeld N, Lee HK, Cazacu S, Finniss S, Xiang C, Poisson L, de Carvalho AC, Slavin S, Jacoby E, Yalon M, Toren A, Mikkelsen T, Brodie C: MicroRNA-137 is downregulated in glioblastoma and inhibits the stemness of glioma stem cells by targeting RTVP-1. Oncotarget 2013;4:665-676.

12 Wiklund ED, Gao S, Hulf T, Sibbritt T, Nair S, Costea DE, Villadsen SB, Bakholdt V, Bramsen JB, Sørensen JA, Krogdahl A, Clark SJ, Kjems J: MicroRNA alterations and associated aberrant DNA methylation patterns across multiple sample types in oralsquamous cell carcinoma. PLoS One 2011;6:e27840.

13 Zhu X, Li Y, Shen H, Li H, Long L, Hui L, Xu W: miR-137 inhibits the proliferation of lung cancer cells by targeting CDC42 and Cdk6. FEBS Lett 2013;587:73-81.

14 Balaguer F, Link A, Lozano JJ, Cuatrecasas M, Nagasaka T, Boland CR, Goel A: Epigenetic silencing of miR137 is an early event in colorectal carcinogenesis. Cancer Res 2010;70:6609-6618.

15 Liang L, Li X, Zhang X, Lv Z, He G, Zhao W, Ren X, Li Y, Bian X, Liao W, Liu W, Yang G, Ding Y: MicroRNA-137, an HMGA1 target, suppresses colorectal cancer cell invasion and metastasis in mice by directly targeting FMNL2. Gastroenterology 2013;144:624-635.

16 Chen Q, Chen X, Zhang M, Fan Q, Luo S, Cao X: miR-137 is frequently down-regulated in gastric cancer and is a negative regulator of CDC42. Dig Dis Sci 2011;56:2009-2016.

17 Smrt RD, Szulwach KE, Pfeiffer RL, Li X, Guo W, Pathania M, Teng ZQ, Luo Y, Peng J, Bordey A, Jin P, Zhao $\mathrm{X}$ : MicroRNA miR-137 regulates neuronal maturation by targeting ubiquitin ligase mind bomb-1. Stem Cells 2010;28:1060-1070.

18 Boyer LA, Lee TI, Cole MF, Johnstone SE, Levine SS, Zucker JP, Guenther MG, Kumar RM, Murray HL, Jenner RG, Gifford DK, Melton DA, Jaenisch R, Young RA: Core transcriptional regulatory circuitry in human embryonic stem cells. Cell 2005;122:947-956.

19 Jiang K, Ren C, Nair VD: MicroRNA-137 represses Klf4 and Tbx3 during differentiation of mouse embryonic stem cells. Stem Cell Res 2013;11:1299-1313.

-20 Sun G, Ye P, Murai K, Lang MF, Li S, Zhang H, Li W, Fu C, Yin J, Wang A, Ma X, Shi Y: miR-137 forms a regulatory loop with nuclear receptor TLX and LSD1 in neural stem cells. Nat Commun 2011;2:529.

-21 Szulwach KE, Li X, Smrt RD, Li Y, Luo Y, Lin L, Santistevan NJ, Li W, Zhao X, Jin P: Cross talk between microRNA and epigenetic regulation in adult neurogenesis. J Cell Biol 2010;189:127-141.

22 Zuk PA, Zhu M, Mizuno H, Huang J, Futrell JW, Katz AJ, Benhaim P, Lorenz HP, Hedrick MH: Multilineage cells from human adipose tissue: implications for cell-based therapies. Tissue Eng 2001;7:211-228.

-23 Zuk PA, Zhu M, Ashjian P, De Ugarte DA, Huang JI, Mizuno H, Alfonso ZC, Fraser JK, Benhaim P, Hedrick MH: Human adipose tissue is a source of multipotent stem cells. Mol Biol Cell 2002;13:4279-4295.

24 Rodriguez AM, Elabd C, Amri EZ, Ailhaud G, Dani C: The human adipose tissue is a source of multipotent stem cells. Biochimie 2005;87:125-128.

25 Gimble JM, Guilak F: Differentiation potential of adipose derived adult stem (ADAS) cells. Curr Top Dev Biol 2003;58:137-160.

26 Kim YJ, Bae SW, Yu SS, Bae YC, Jung JS: miR-196a regulates proliferation and osteogenic differentiation in mesenchymal stem cells derived from human adipose tissue. J Bone Miner Res 2009;24:816-825.

27 Kim YJ, Hwang SH, Cho HH, Shin KK, Bae YC, Jung JS: MicroRNA 21 regulates the proliferation of human adipose tissue-derived mesenchymal stem cells and high-fat diet-induced obesity alters microRNA 21 expression in white adipose tissues. J Cell Physiol 2012;227:183-193.

28 Kim YJ, Kim HK, Cho HH, Bae YC, Suh KT, Jung JS: Direct comparison of human mesenchymal stem cells derived from adipose tissues and bone marrow in mediating neovascularization in response to vascular ischemia. Cell Physiol Biochem 2007;20:867-876.

-29 Dweep H, Sticht C, Pandey P, Gretz N: miRWalk--database: prediction of possible miRNA binding sites by "walking" the genes of three genomes. J Biomed Inform 2011;44:839-847.

30 Spiegelman BM, Flier JS: Obesity and the regulation of energy balance. Cell 2001;104:531-543.

31 Flier JS: Obesity wars: molecular progress confronts an expanding epidemic. Cell 2004;116:337-350.

-32 Tontonoz P, Hu E, Graves RA, Budavari AI, Spiegelman BM: mPPAR gamma 2: tissue-specific regulator of an adipocyte enhancer. Genes Dev 1994;8:1224-1234.

-33 Lane MD, Tang QQ, Jiang MS: Role of the CCAAT enhancer binding proteins (C/EBPs) in adipocyte differentiation. Biochem Biophys Res Commun 1999;266:677-683.

-34 Rosen ED, Sarraf P, Troy AE, Bradwin G, Moore K, Milstone DS, Spiegelman BM, Mortensen RM: PPAR gamma is required for the differentiation of adipose tissue in vivo and in vitro. Mol Cell 1999;4:611-617. 
-35 Patel KJ, Pambuccian SE, Ondrey FG, Adams GL, Gaffney PM: Genes associated with early development, apoptosis and cell cycle regulation define a gene expression profile ofadenoid cystic carcinoma. Oral Oncol 2006;42:994-1004

-36 Cappello S, Attardo A, Wu X, Iwasato T, Itohara S, Wilsch-Bräuninger M, Eilken HM, Rieger MA, Schroeder TT, Huttner WB, Brakebusch C, Götz M: The Rho-GTPase CDC42 regulates neural progenitor fate at the apical surface. Nat Neurosci 2006;9:1099-1107.

- 37 Wu X, Quondamatteo F, Lefever T, Czuchra A, Meyer H, Chrostek A, Paus R, Langbein L, Brakebusch C: CDC42 controls progenitor cell differentiation and beta-catenin turnover in skin. Genes Dev 2006;20:571-585.

38 Yang L, Wang L, Kalfa TA, Cancelas JA, Shang X, Pushkaran S, Mo J, Williams DA, Zheng Y: CDC42 critically regulates the balance between myelopoiesis and erythropoiesis. Blood 2007;110:3853-3861.

39 Sordella R, Jiang W, Chen GC, Curto M, Settleman J: Modulation of Rho GTPase signaling regulates a switch between adipogenesis and myogenesis. Cell 2003;113:147-158.

40 McBeath R, Pirone DM, Nelson CM, Bhadriraju K, Chen CS: Cell shape, cytoskeletal tension, and RhoA regulate stem cell lineage commitment. Dev Cell 2004;6:483-495.

41 Bryan BA, Mitchell DC, Zhao L, Ma W, Stafford LJ, Teng BB, Liu M: Modulation of muscle regeneration, myogenesis, and adipogenesis by the Rho family guanine nucleotide exchange factor GEFT. Mol Cell Biol 2005;25:11089-11101.

42 Nishizuka M, Arimoto E, Tsuchiya T, Nishihara T, Imagawa M: Crucial role of TCL/TC10beta L, a subfamily of Rho GTPase, in adipocyte differentiation. J Biol Chem 2003;278:15279-15284.

43 Philips A, Roux P, Coulon V, Bellanger JM, Vié A, Vignais ML, Blanchard JM: Differential effect of Rac and CDC42 on p38 kinase activity and cell cycle progression of nonadherent primary mouse fibroblasts. J Biol Chem 2000;275:5911-5917.

-44 Schlegel N, Meir M, Spindler V, Germer CT, Waschke J: Differential role of Rho GTPases in intestinal epithelial barrier regulation in vitro. J Cell Physiol 2011;226:1196-1203.

45 Van Hennik PB, Hordijk PL: Rho GTPases in hematopoietic cells. Antioxid Redox Signal 2005;7:1440-1455. 